\title{
Development of an ontology-based semantic building post-occupancy evaluation framework
}

\author{
Yuanhong Zhao ${ }^{1,2, *}$ (D) and Qingping Yang ${ }^{2}$ \\ ${ }^{1}$ Department of Civil and Environmental Engineering, Brunel University London, London, UK \\ ${ }^{2}$ Quality Engineering and Smart Technology Research Centre, College of Engineering, Design and Physical Sciences, Brunel \\ University London, London, UK
}

Received: 31 March 2021 / Accepted: 8 July 2021

\begin{abstract}
Post-occupancy evaluation (POE) is a systematic method to evaluate the actual building performance against the theoretical design intents after the building has been occupied for some time, to understand how the building is performing and to capture lessons learned. The POE offers an opportunity to investigate the buildings' actual performance based upon the occupants' satisfaction levels in the aspects of building overall design, indoor environmental quality, thermal comfort, etc. However, as the key part of POE, occupant satisfaction assessment (OSA) is a missing link in the building performance evaluation (BPE) domain, and there is not a systematic evaluation method for the OSA. Moreover, it is time-consuming and error-prone to conduct the OSA manually. This paper presents from the end-user's satisfaction perspective a semantic postoccupancy evaluation ontology (POEontology) to facilitate the occupant satisfaction assessment of buildings, with the ultimate aim of optimizing building operation guidelines, and improving occupants' use experience quality and well-being. An ontology-based knowledge model has been developed to capture the fragmented knowledge of building use satisfaction assessment in the POE domain, with the benchmarking evaluation rules encoded in Semantic Web Rule Language (SWRL) to enable automatic rule-based rating and reasoning. This ontology model also enables the effective OSA-related knowledge retrieving and sharing, and promotes its implementation in the POE domain. A field study has been conducted based upon the Building Use Study (BUS) methodology to validate the proposed ontology framework.
\end{abstract}

Keywords: Occupant satisfaction assessment (OSA) / post-occupancy evaluation (POE) / ontology / semantic web rule language (SWRL) / reasoning and querying / building use study (BUS) methodology

\section{Introduction}

Post-occupancy evaluation (POE) is a systematic method for measuring the actual building performance in meeting design intents, and identifying the performance gaps between actual performance and the standard criteria in indoor environment quality (IEQ), building design, occupant satisfaction, productivity, energy consumption, etc. [1]. The POE is essential for measuring the functional performance of current buildings and improving the operation of future buildings [2]. The value of POE implementation is being increasingly recognized and hundreds of POEs have been performed in different fields. In the Post-occupancy Review of Buildings and their Engineering (PROBE) study, which started in 1995, led by the UK government and a research team to assess the performance of commercial and public buildings, the

\footnotetext{
* Corresponding author: yuanhong.zhao@brunel.ac.uk
}

evaluation results have shown the occupants had low satisfaction with their buildings. The USGBC (U.S. Green Building Council) reports that it is important to consider occupant satisfaction after the building is finished and the offices are occupied by bookshelves, computers, furniture and people. The PROBE study's authors substantiated the importance of feedback when they stated that "managed feedback produces better buildings" [3].

Another recent UK government led BPE (Building Performance Evaluation) project involving 56 buildings in 2016 has shown that even the BREEAM (Building Research Establishment Environmental Assessment Method) certificated office buildings were not performing as they should do, the buildings consuming up to 3-10 times the energy specified, and some of the end-users have lower satisfaction compared with the non-certificated buildings [4]. More recently, BSRIA (Building Services Research and Information Association) has launched the six-phase approach named Soft Landing Framework to raise awareness of building performance in use, Phase 6 
emphasizes and provides an effective route for the aftercare and POE of buildings [5]. Khair et al. [6] conducted a study by using POE tools to assess the physical environment elements of public low-cost housings based on occupants' preferences in Malaysian. Alborz [7] developed a POE framework for the evaluation of sustainable dormitories in higher education and established the evaluation criteria hierarchy, but this framework is not suitable for other building types, like office buildings. The POE in China had a late start, the government has proposed the first national standard of the "Technical Manual of Post-occupancy Evaluation for Green Building" in 2017 to promote the POE practice among the green building [8].

Building performance evaluation (BPE) originated from England and the United States, by the early work from Manning and Markus et al. [9] and it has been applied in different forms since 1960s. Along with the later development, post-occupancy evaluation (POE) has become one of the most widely applicable and sustained methods as a sub-process of BPE.

As a systematic method, the POE has been expanding in research to covers many assessment indicators in the aspects of energy, environment, and occupant comfort; it provides feedback to optimize and improve building performance throughout the whole building lifecycle, from the stage of strategic planning, design, construction, occupation to operation, and typically including analysis of the end user's perceptions [10].

Unlike other building performance evaluation systems, POE promotes the participation of building occupants, the end-users, focusing on their requirements of buildings in the aspects of health, safety, convenience, amenity, psychological comfort, living quality and satisfaction [11]. In BS 8536-1:2015 [12], POE is defined as "process of evaluating an asset/facility after it has been completed and is in use to understand its actual performance against that required and to capture lessons learned". Blyth, Gilby and Barlex [13] defined POE as a way to provide the performance feedback throughout the whole lifecycle of buildings, from the initial concept design, construction, operation and maintenance to occupation. The feedback from the POE evaluation can help to provide development guidance for future similar projects. It can not only provide the feedback on building performance, but also produce positive impacts on running costs, occupants' well-being and business efficiency (BRE).

In general, it can be seen as a broad range of activities aimed at understanding how buildings perform through the life cycle assessment and how satisfied building users are with the physical and environmental factors, the main features to be evaluated should include users' satisfaction, their assessment of building comfort and function, as well as their behaviours [14].

An understanding of the impacts of occupant interactions with the building and the outcomes in terms of comfort, health and satisfaction are critical elements and the methods to evaluate these have continued to evolve [15]. However, the majority of POE studies contributed to revealing building performance gaps in energy use and resource consumption through the building monitoring, the information like occupants satisfaction cannot be obtained by such quantitative methods [16], with emerging needs in improving end-user's experience quality, the implementation of $\mathrm{POE}$ in end-user satisfaction has become significant, understanding the end-user satisfaction is the key factor to improve the occupants' living experience quality.

At the same time, there has been no specific systematic evaluation method for occupant satisfaction evaluation, and it is time-consuming and error-prone to conduct the assessment manually. This research utilized ontology as a semantic web technology to represent the assessment knowledge in the domain of occupant satisfaction POE.

A comprehensive POE method, one that includes assessments of occupant well-being and productivity, completes the feedback loop that is essential for the successful future development and improvement of building design and practices. To make up the missing link in the POE domain, this paper has presented a semantic postoccupancy evaluation ontology (POE ontology) from the end-user's satisfaction perspective, to facilitate the occupant satisfaction assessment of buildings and to promote the knowledge sharing, with the ultimate aims of optimizing building operation guidelines, and improving occupants' use experience quality and well-being.

As an extension of current World Wide Web, the Semantic Web coined by Tim Berners-Lee [17] allows the web data to have explicit meaning and to be interpreted by machines, achieving the interoperability between human and machine. Compared with the current Web, the Semantic Web makes it easier to automatically process, integrate and interpret information, similar to the way humans process information. The Semantic Web provides highly efficient technologies, such as Resource Description Framework (RDF), RDF Schema (RDFS), Web Ontology Language (OWL) and SPARQL to formally represent semantic data.

Ontology is one of the core Semantic Web technologies used to describe concepts, terms, relationships between entities within a given knowledge domain. In philosophy, the term ontology refers to the science that describes the nature of being and their relations. In computer and information science, ontology has been widely used in the domain of knowledge representation, sharing and management by conceptualizing the knowledge concepts and their relations. An ontology may be defined as 'an explicit specification of a conceptualization' [18]. Uschold and Gruninger [19] have pointed out that the ontology is a term referring to the shared understanding of a given domain, and it can be used as a unifying problem-solving framework. Ontology is a formal and declarative knowledge representation system, the terms related to the subject domain and the logical relationship statements between the terms are declared in this system. Noy and McGuinness [20] characterised ontology as a common domain vocabulary, which defines the domain knowledge or information concepts and clarifies their relations to facilitate communication among domain experts, and achieve the communication between the domain experts and knowledge-based systems, and their Simple-Knowledge Engineering Methodology (SKEM) method has been widely adopted to develop the ontology in different domains, this method is also known as the 'Seven-Step' method. 
The ontology technology has been used in many different domains, for example, adopting the SWRL method to express and execute the complex product design process in the industry [21], the energy management in buildings [22], knowledge management and retrieval system [23], biomedical domain [24], and so on. As an advanced semantic technique, ontology has also been widely used in the building design, construction, operation, maintenance, and facility management domains [25]. In this research ontology has been adopted as an effective way to develop a framework to support the occupant satisfaction assessment in the POE, as well as relevant knowledge reusing and sharing.

There have been some existing ontologies developed to support knowledge representation and management within the building evaluation domain. For example, Construction Quality Inspection and Evaluation Ontology (CQIE Ontology) is developed against manual construction quality compliance checking, which is time-consuming, cumbrous and error-prone. It enables the construction quality checking to be carried out as a concurrent activity along with the construction process, rather than afterward [26]. However, this ontology mainly focuses on the quality checking during the construction stage, it does not cover the occupant satisfaction assessment of POE. Sustainable Building Technology (SBT) Ontology explains the three main concepts in the SBT domain to represent the knowledge about the emerging sustainable building technologies in UK construction, including building construction technology, organization and the standards required for the design of construction. Each of these three concepts consists of different subclasses [27]. The eeBIMontology (Energy Enhanced BIM) framework is used for the building energy performance analysis, and facilitates to identify the energy performance problems at the early design stage [28]. However, this ontology framework focuses more on building energy performance simulations at the early design stage, not suitable for the total building performance evaluation of the whole building lifecycle. Boje [29] proposed a method to integrate ontology as a knowledge representation technique into a BIM supported building fire evacuation design process to facilitate automatic evacuation design decision-making and improve the interoperability of the BIM system. Zhang et al. [30] proposed an intelligent green building rating (iGBR) framework supported by a semantic and social approach to realize real-time rating in building design stage, and to provide designers with the ability to evaluate the sustainability level of a building project against a given GBRS in a real-time design process. Ding et al. developed an ontology-based framework to facilitate the construction risk knowledge management in BIM environment [31].

There are some other representative ontologies in the architecture, engineering and construction (AEC) industry knowledge management field, such as the CSC Ontology for construction safety checking [32], the CNC Ontology for construction noise control [33], the Think Home for energy efficiency in future smart homes [34], the green building post-occupancy evaluation assessment knowledge modelling ontology, which is a knowledge representation model based upon a Chinese POE assessment standard and is only a knowledge model without rules and queries, focusing on the domain of green building labelling evaluation [35], the Dog Ont ontology used to represent energy-related information [36], and so on. These ontologies are specialized in different AEC industry domains. However, there is a missing ontology model for the building occupant satisfaction domain.

By integrating the ontology, SWRL rules, and BUS methodology, this paper has proposed an enhanced semantic building POE framework, which facilitates measuring the building occupant satisfaction. At first, the assessment knowledge and evaluation SWRL rules are systematically constructed into an ontology model, which is the critical foundation of the proposed assessment framework. Then, based on the pre-defined rules to execute the reasoning process and querying to generate the automatic assessment score results. Finally, based on the assessment results to optimise the building operation with the ultimate goal of improving the building operation quality. The following section will detail the proposed ontology and its development methodology and process.

\section{Framework development}

\subsection{Overview}

As shown in Figure 1, an ontology-based framework has been developed to capture the fragmented building use satisfaction assessment in the POE domain, the benchmarking evaluation rules are encoded in SWRL to enable automatic rule-based scoring and reasoning. This ontology model also enables the effective OSA-related knowledge retrieving and sharing, and promotes its implementation in the POE domain. A field study is conducted based on the Building Use Study (BUS) methodology to verify the proposed ontology framework. The required data for this evaluation is extracted from the field BUS survey, combined with SWRL rules from the ontology and exported to the reasoning engine.

The proposed framework involves the main three steps of the evaluation process: ontology model development, ontology assessment application, building operation feedback and optimization.

- Ontology model development: this is the core step of the framework development, including knowledge extraction, survey data collection and input, and assessment rules editing. This research uses the knowledge management system Protégé to construct the ontology model. The assessment knowledge is extracted from the BUS methodology standard to enumerate core concepts and define their properties and values. The BUS methodology is applied to conduct a building survey, then the collected data is transferred as the values of instances into the ontology model, and then evaluation principles are transferred into SWRL rules to set the assessment constraints for Michael Sterling ontology applications.

- Ontology assessment application: rules-based reasoning and querying. Based on the edited ontology knowledge and rules in Step 1, the new knowledge is generated in rule engine after the logical reasoning, and the evaluation can be conducted using the querying rules. 


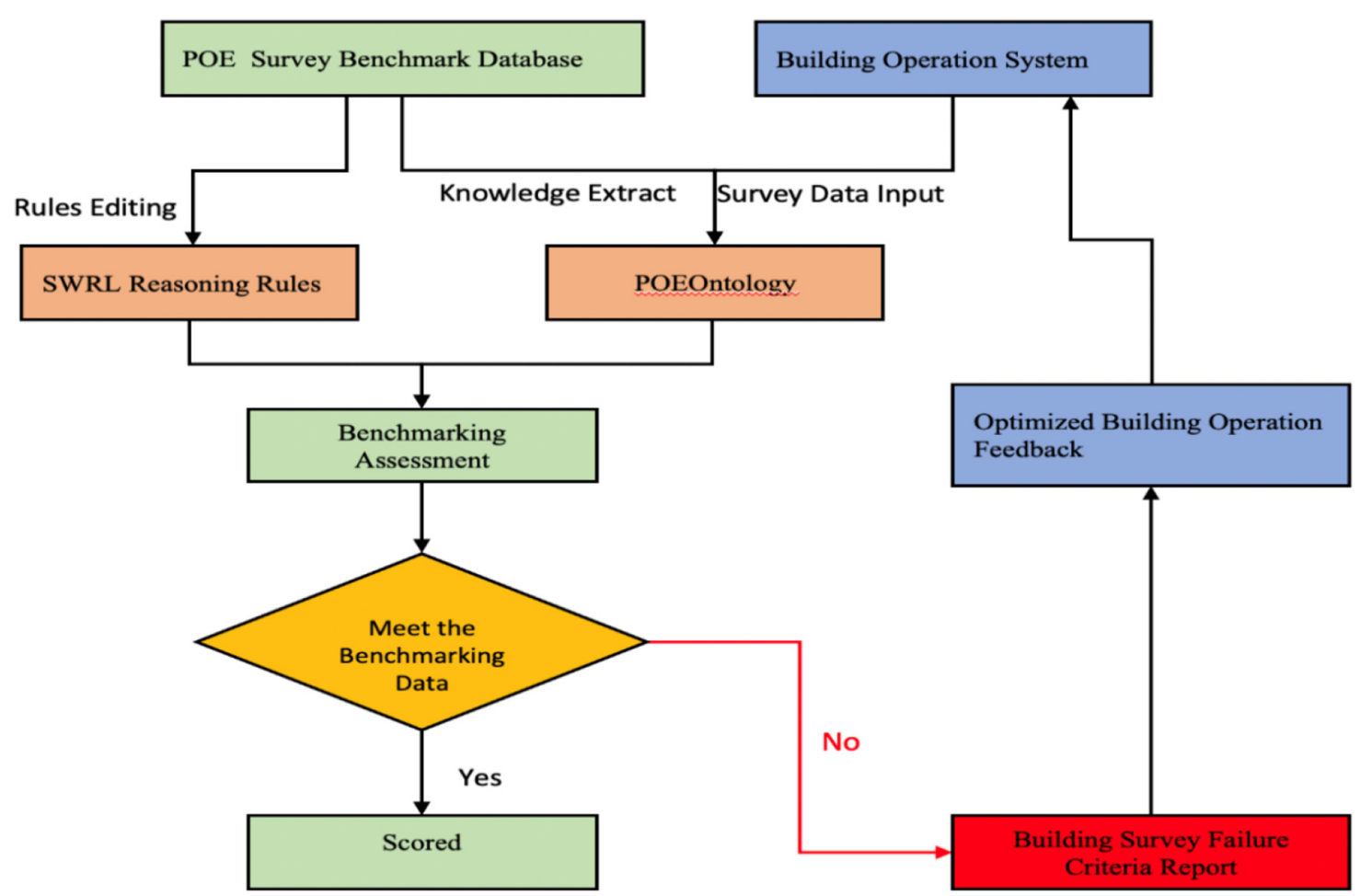

Fig. 1. The framework of comprehensive post occupancy building performance evaluation.

Table 1. Ontology development methodologies.

\begin{tabular}{|c|c|c|}
\hline Methodology & Time & Development processes \\
\hline Uschold and King's methodology & 1995 & $\begin{array}{l}\text { Identify the purpose and scope of ontology } \rightarrow \\
\text { Ontology building (ontology capture, coding, } \\
\text { integrating existing ontology) } \rightarrow \text { Evaluation } \rightarrow \\
\text { Documentation }\end{array}$ \\
\hline Grüninger and Fox's Methodology & 1995 & $\begin{array}{l}\text { Motivating Scenarios } \rightarrow \text { Informal Competency } \\
\text { Questions }(\mathrm{CQ}) \rightarrow \text { Formal Terminology } \rightarrow \\
\text { Formal CQ } \rightarrow \text { Formal Axioms } \rightarrow \text { Completeness } \\
\text { Theorems }\end{array}$ \\
\hline METHONTOLOGY & 1997 & $\begin{array}{l}\text { Planning } \rightarrow \text { Specification } \rightarrow \text { Knowledge } \\
\text { Acquisition } \rightarrow \text { Conceptualization } \rightarrow \\
\text { Formalization } \rightarrow \text { Integration } \rightarrow \text { Implementation } \rightarrow \\
\text { Evaluation } \rightarrow \text { Documentation } \rightarrow \text { Maintaining }\end{array}$ \\
\hline $\begin{array}{l}\text { Simple-Knowledge Engineering Methodology (SKEM)/ } \\
\text { Seven-Step Method }\end{array}$ & 2001 & $\begin{array}{l}\text { Determine the domain and scope } \rightarrow \text { Consider } \\
\text { reusing existing ontologies } \rightarrow \text { Enumerate import } \\
\text { terms } \rightarrow \text { Define the classes } \rightarrow \text { Define the slots } \rightarrow \\
\text { Define the facets of the slots } \rightarrow \text { Create instances }\end{array}$ \\
\hline
\end{tabular}

- Building operation feedback and optimization: according to the evaluation outcomes feedback from Step 2 to provide the building operation feedback and optimization suggestions.

\subsection{Ontology development methodologies}

There is no single correct methodology to design an ontology for any given domain. There are a few ontology-design methods, for example, Grüninger and Fox's methodology, Uschold and King's method, METHONTOLOGY, and Simple-Knowledge Engineering Methodology (SKEM) which is also named as the 'SevenStep' Method and is the most prevailing one. These ontology building methodologies and their development processes are listed in Table 1. Catering to improve ontology's expressivity capability, the W3C developed several ontology description languages, including XML, RDF, RDFS and OWL. Because of the ability to represent 


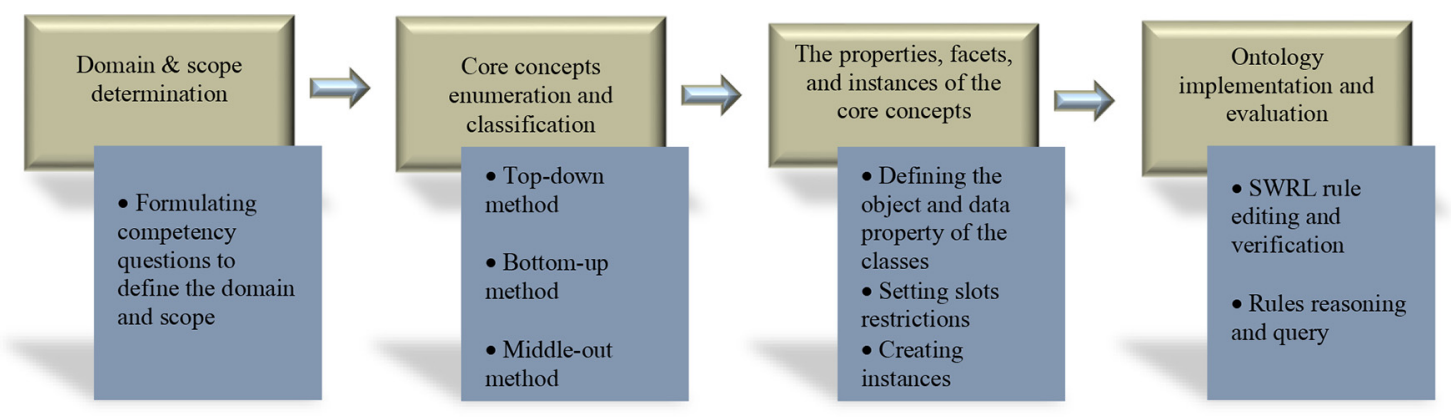

Fig. 2. The 'Seven-Step' method of ontology development.

Table 2. The class hierarchies of some ontology terms.

\begin{tabular}{lll}
\hline Class hierarchy & Superclass & Sub-criteria \\
\hline & EvaluationCriteriaConditionsInSummer & CriteriaAirQualityInSummer \\
EvaluationCriteria & CriteriaTemperatureInSumme \\
& EvaluationCriteriaLighting & CriteriaAmountOfArtificialLight \\
& & CriteriaGlareFromLights \\
& EvaluationTaskConditionsInSummer & TaskAirQualityInSummer \\
EvaluationTask & & TaskTemperatureInSummer \\
& EvaluationTaskLighting & TaskAmountOfArtificialLight \\
& & TaskGlareFromLights \\
BuildingType & Domestic & - \\
Evaluator & NonDomestic & - \\
\hline
\end{tabular}

rich and complex knowledge and reasoning ability, OWL is recommended by $\mathrm{W} 3 \mathrm{C}$ as a proper ontology description language to be used in ontology development. There are several available ontology editors, e.g. OntoEdit, WebODE Swoop, OntoStudio and Protégé. Protégé is a free, open-source, user-friendly ontology editor and framework with a suite of tools for building intelligent systems, and also the most widely used one.

By adopting the 'Seven-Step' methodology, the simplified process of the comprehensive ontology development method is shown in Figure 2.

Different from the initial 'Seven-Step' methodology, this research has integrated the ontology querying and reasoning part into the ontology development process, making the rule editing as part of the ontology building.

From end-user's satisfaction perspective, this research proposes an ontology knowledge model to facilitate the occupant satisfaction assessment of buildings, aiming to optimize building operation and achieving higher building user experience quality. The proposed ontology model includes the concepts extracted from the evaluation systems and relevant researches. In the core concepts enumeration and classification step, this research has adopted the middle-out methodology, a mix of top-down and bottom-up methods, proposed by Uschold and Gruninger [18] to list important terms, and the terms hierarchy relations are shown in Table 2 .

By following the classification principle that if class $\mathrm{A}$ is a superclass of class B, then every single instance of class B is also an instance of class $\mathrm{A}$, so the relationship between $\mathrm{A}$ and $\mathrm{B}$ is a 'kind of' relation. The following Figure 3 illustrates all the asserted class hierarchies in the proposed model. The selected top superclass are conditions in summer, conditions in winter, control, lighting, noise, building overall, and so on. Under each of the top criteria, there are some sub-criteria, for example, the sub-criteria of conditions in summer are air movement, air quality, temperature, humidity, ventilation and temperature variation. The sub-criteria of lighting are amount of artificial light and natural light, the glare from lights and sun.

The internal relations of concepts are defined as the object property of classes, and the relations between concepts and their values are defined as data property as shown in Figures 4 and 5. Every assessment criterion has both lower and upper thresholds values, which means every single criterion has two data properties; the lower critical limit value, the upper critical limit value, so these data properties are described in the form of hasLowerCritical- 


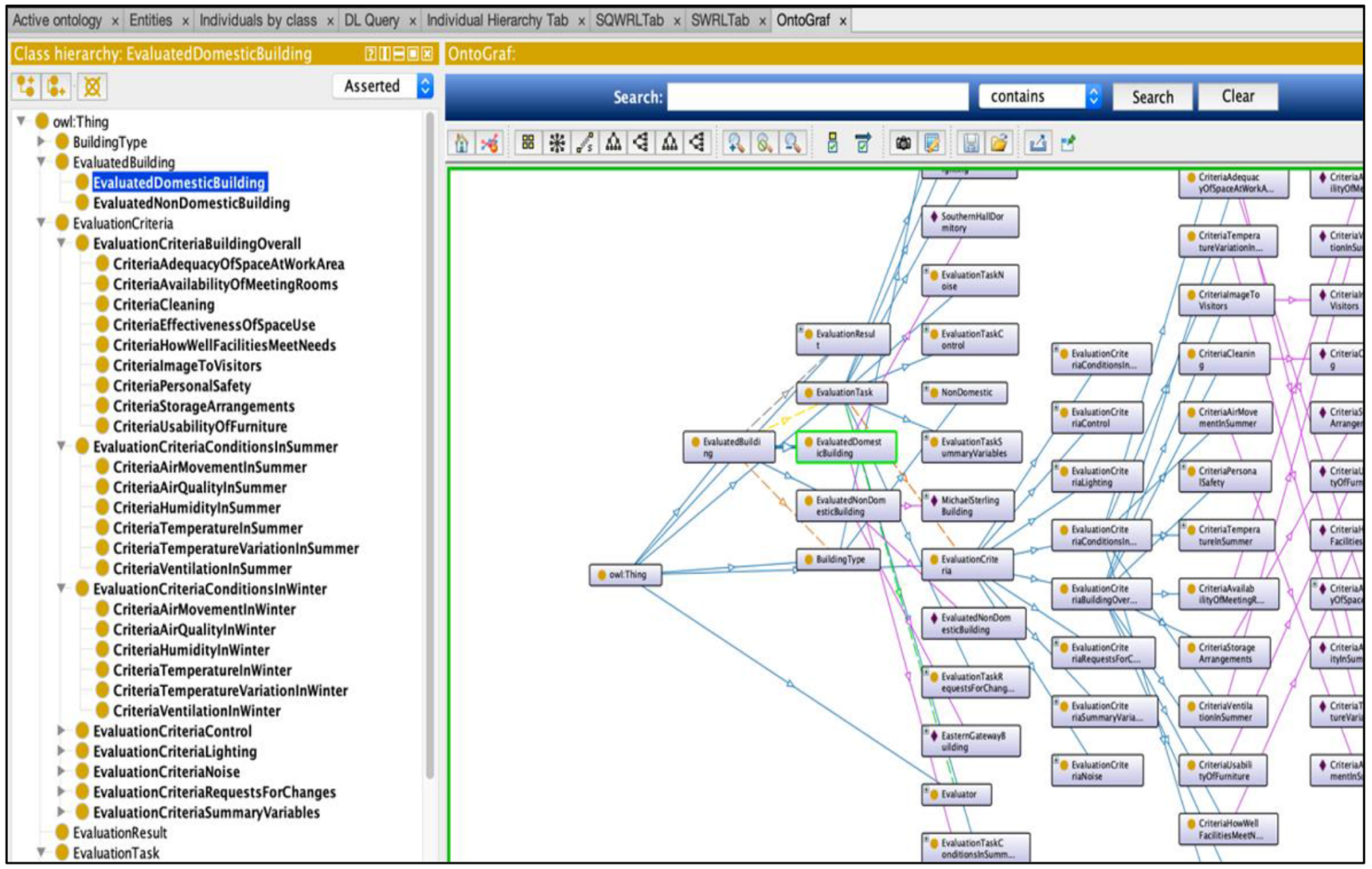

Fig. 3. The proposed ontology model.

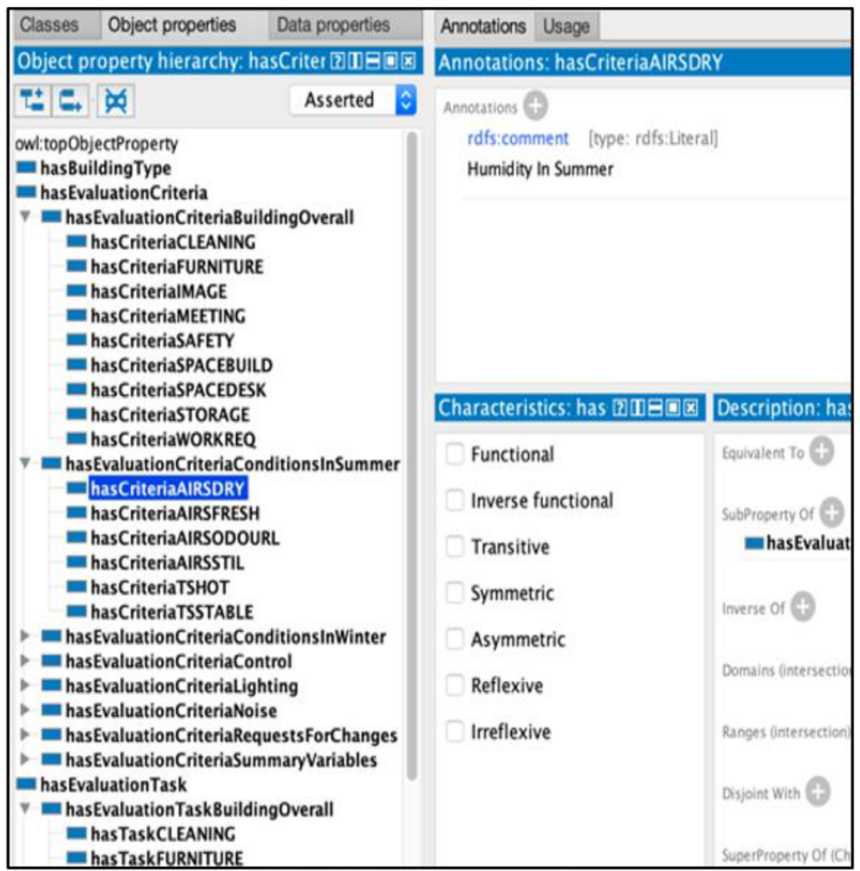

(a)

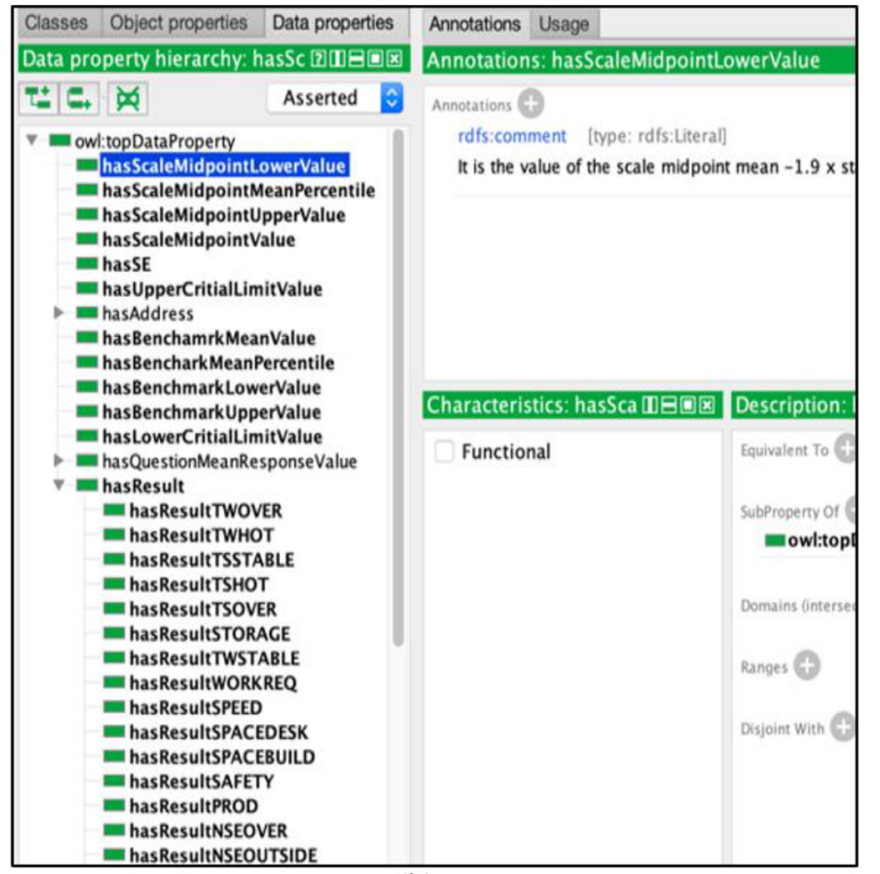

(b)

Fig. 4. Object property and data property in the proposed ontology. (a) objective property. (b) data property. 


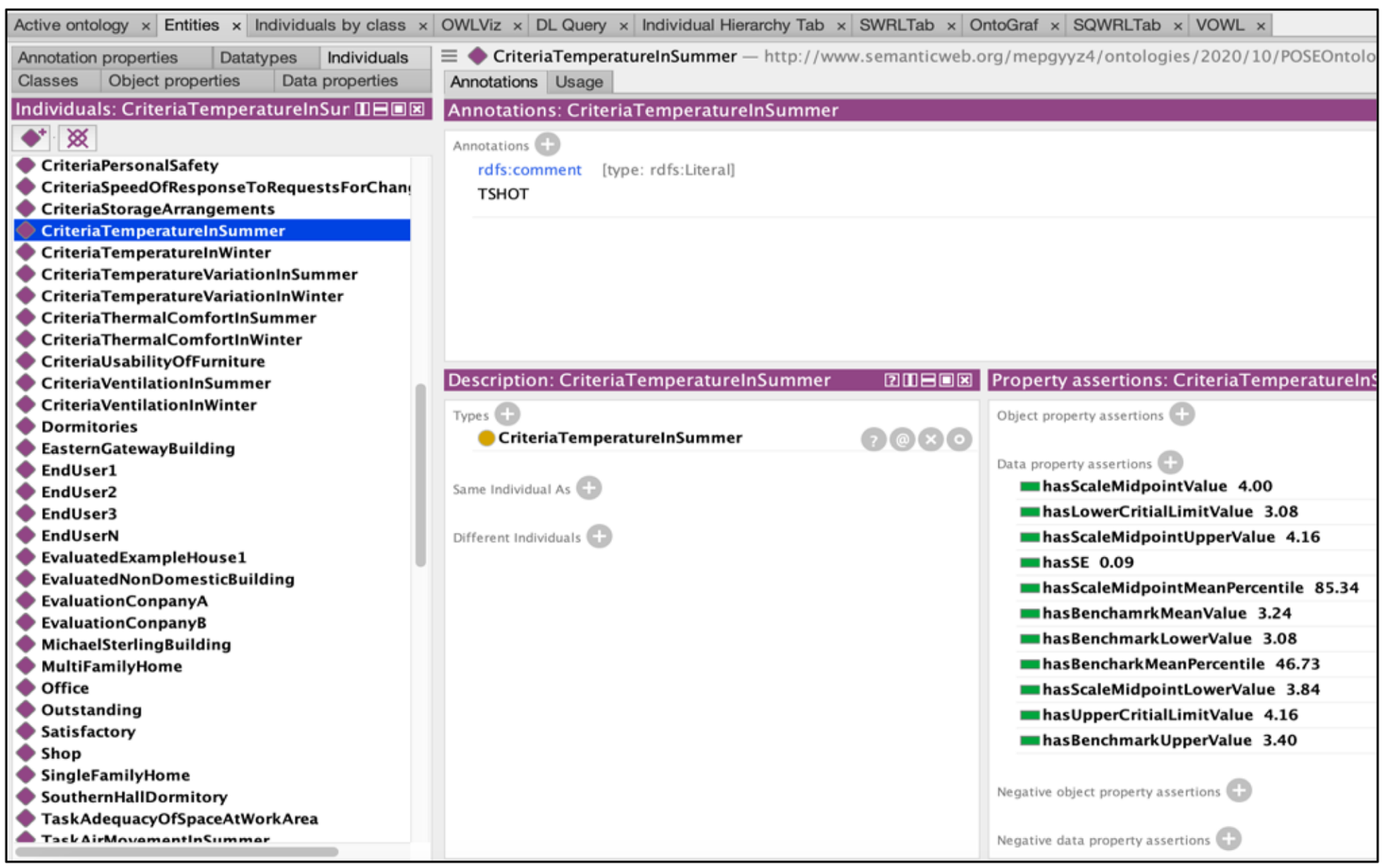

Fig. 5. The individuals of proposed ontology.

LimitValue and hasUpperCriticalLimitValue. For example, the lower critical limit value of assessment criteria temperature in summer is 3.08, so the expression in ontology model is CriteriaTemparatureInSummer hasLowerCriticalLimitValue 3.08. Here, the data values are the facets of the CriteriaTemperatureInSummer slot, as each evaluation criteria can have only one exact critical value, so the slot restrictions of CriteriaTemperatureInSummer has exactly 1 critical value for each data property, and the value is a float type. The data property values of the created instances of classes are shown in Figure 5.

\subsection{Rule-based reasoning using SWRL}

The Semantic Web Rule Language (SWRL) is a standard ontological rule language for the Semantic Web that is used to express rules and logic, it is developed based on the OWL-DL and the Rule Markup Language (Rule ML). A SWRL rule is in the form of an implication between an antecedent (body) and a consequent (head) that are connected by implication symbol ' $\rightarrow$ ', and it is written in a human readable syntax as: antecedent $\rightarrow$ consequent, which can be read as: if the condition specified in the antecedent applies, then the condition specified in the consequent must also hold. Both of the antecedent (body) and the consequent (head) consist of a conjunction of one or more atoms, combined by symbol ' $\wedge$ '. There are seven types of atoms provided by SWRL: class atoms, individual property atoms, data valued property atoms, different individual atoms, same individual atoms, built-in atoms and data range atoms [37].

In this syntax, a rule has the form:

$$
\text { antecedent }->\text { consequent }
$$

where the antecedent and consequent consist of conjunction of atoms, as expressed in equation (2):

$$
\begin{aligned}
& \underset{\mathrm{atom}}{\mathrm{a} 1} \Lambda \operatorname{atom}_{\mathrm{a} 2} \Lambda \cdots \operatorname{atom}_{\mathrm{an}} \\
& \quad \rightarrow \operatorname{atom}_{c 1} \Lambda \operatorname{atom}_{c 2} \Lambda \cdots \operatorname{atom}_{c m}
\end{aligned}
$$
OWL:

As shown in equation (3), every atom is a predicate in

$$
p\left(\arg _{1}, \arg _{2} \ldots \arg _{n}\right)
$$

where $p$ is a predicate symbol defined in OWL and $\arg _{1}$, $\arg _{2} \ldots \arg n$ are the expression of specific terms or parameters. The form of atom can be $C(x), P(x, y)$, sameAs $(x, y)$ or differentFrom $(x, y)$, where $\mathrm{C}$ is an $\mathrm{OWL}$ classes description, $\mathrm{P}$ represents the OWL properties, and $\mathrm{x}, \mathrm{y}$ are either variables, OWL individuals or OWL data values, whilst sameAs $(x, y)$ or differentFrom $(x, y)$ are the built-in functions of SWRL [38]. 
Table 3. Examples of SWRL rules.

\begin{tabular}{|c|c|}
\hline Rule & Description \\
\hline \multicolumn{2}{|c|}{ Temperature in Winter (tiw) } \\
\hline 1 & $\begin{array}{l}\text { EvaluatedNonDomesticBuilding(?endb) } \wedge \\
\text { hasQuestionMeanResponseValueTWHOT (?endb, ?qmrvtwhot })^{\wedge} \\
\left.\text { CriteriaTemperatureInWinter(?ctiw })^{\wedge} \text { hasLowerCritialLimitValue(?ctiw, ?lclv }\right)^{\wedge} \\
\left.\text { swrlb:lessThan(?qmrvtwhot, ?lclv) }{ }^{\wedge} \text { TaskTemperatureInWinter(?ttiw }\right)^{\wedge} \\
\text { hasCriteriaTWHOT(?ttiw, ?ctiw })^{\wedge} \text { hasTaskTWHOT(?endb, ?ttiw) }-> \\
\text { hasResultTWHOT(?endb, Too Hot) }\end{array}$ \\
\hline 2 & 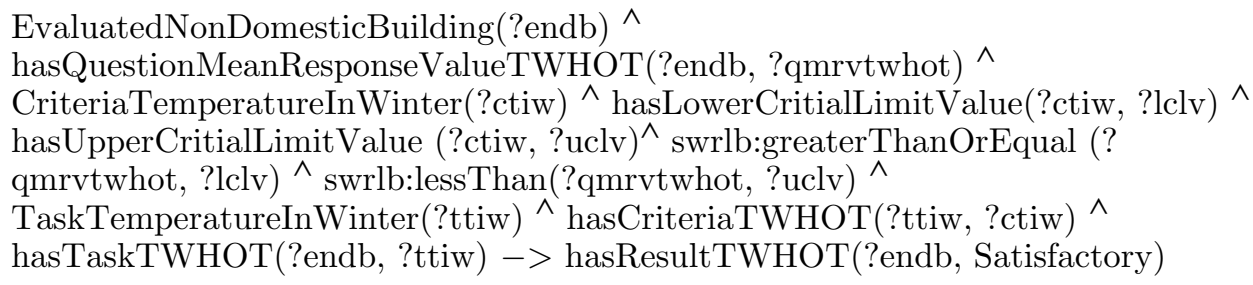 \\
\hline 3 & $\begin{array}{l}\text { EvaluatedNonDomesticBuilding }(\text { ?endb }) \wedge \\
\text { hasQuestionMeanResponseValueTWHOT(?endb, ?qmrvtwhot }) \wedge \\
\text { CriteriaTemperatureInWinter(?ctiw } \wedge \wedge \text { hasLowerCritialLimitValue(?ctiw, ?lclv })^{\wedge} \\
\text { swrlb:greaterThan(?qmrvtwhot, ?lclv) } \wedge \text { TaskTemperatureInWinter(?ttiw }) \wedge \\
\left.\text { hasCriteriaTWHOT(?ttiw, ?ctiw } \wedge^{\wedge} \text { hasTaskTWHOT(?endb, ?ttiw }\right)-> \\
\text { hasResultTWHOT(?endb, Too Cold) }\end{array}$ \\
\hline
\end{tabular}

Air Quality in Summer (aqis) CriteriaAirQualityInSummer(?cairsodourl) ${ }^{\wedge}$ hasLowerCritialLimitValue(? cairsodourl, ?lclv) $\wedge^{\wedge}$ swrlb:lessThan(?qmrvairsodourl, ?lclv) ${ }^{\wedge}$

TaskAirQualityInSummer(?tairsodourl) ^ hasCriteriaAIRSODOURL(? tairsodourl, ?cairsodourl) ${ }^{\wedge}$ hasTaskAIRSODOURL(?endb, ?tairsodourl) $->$ hasResultAIRSODOURL(?endb, Too Smelly) CriteriaAirQualityInSummer(?cairsodourl) ${ }^{\wedge}$ hasLowerCritialLimitValue(? cairsodourl, ?lclv) $\wedge$ swrlb:greaterThanOrEqual(?qmrvairsodourl, ?lclv) ${ }^{\wedge}$ TaskAirQualityInSummer(?tairsodourl) $\wedge$ hasCriteriaAIRSODOURL(? tairsodourl, ?cairsodourl) ${ }^{\wedge}$ hasTaskAIRSODOURL(?endb, ?tairsodourl) $->$ hasResultAIRSODOURL(?endb, Satisfactory)

where $e n d b$ is the evaluated non-domestic building, $q m r v$ is the question mean response value, $l c l v$ is the lower critical limit value, $u c l v$ is the upper critical limit value, TWHOT is temperature in winter hot, ctiw is the criteria temperature in winter, ttiw is the task temperature in winter, aqis is the air quality in summer, AIRSODOURL is the air quality in summer odourless, cairsodourl is the criteria air quality in summer odourless, tairsodourl is the task air quality in summer odourless.

In this research, the ontology editing software Protégé has been used to build the ontology knowledge model, its SWRL tab plugin supports the editing and execution of SWRL rules. The SWRL tab is a SWRL API-based development tool that provides a set of standalone graphical interfaces for managing SWRL rules and SQWRL queries [39].

Following the SWRL syntax, the assessment constraints can be encoded in SWRL rules to perform rulebased reasoning. For example, the occupant satisfaction assessment criteria of air quality in winter and temperature in winter can be coded by the SWRL rules, as shown in Table 3. As shown in Figure 6, the results of temperature in winter may be too hot, too cold, or satisfactory, and the results of air quality in winter assigned different satisfactory levels, either not satisfactory with the assessment result of too smelly, or satisfactory. In non-domestic building, if the occupant satisfaction value is less than the lower criterial limit value, it means the temperature in winter is too hot; if the occupant satisfaction value is between the lower criterial limit value and upper criterial limit value, it means the building occupants are satisfied with the temperature in winter; if the occupant satisfaction value is greater than the upper criterial limit value, is shows that the temperature in winter in this building is too cold. 


\section{Cond/tions in winter overall \\ Figure 7 \\ Adr movement in winter}

Figure 2

Air quality in winter

Figure 3

Humidity in winter

Figure 4

Temperature in winter

Figure 5

Temperature variation in winter

Figure 6

Ventilation in winter

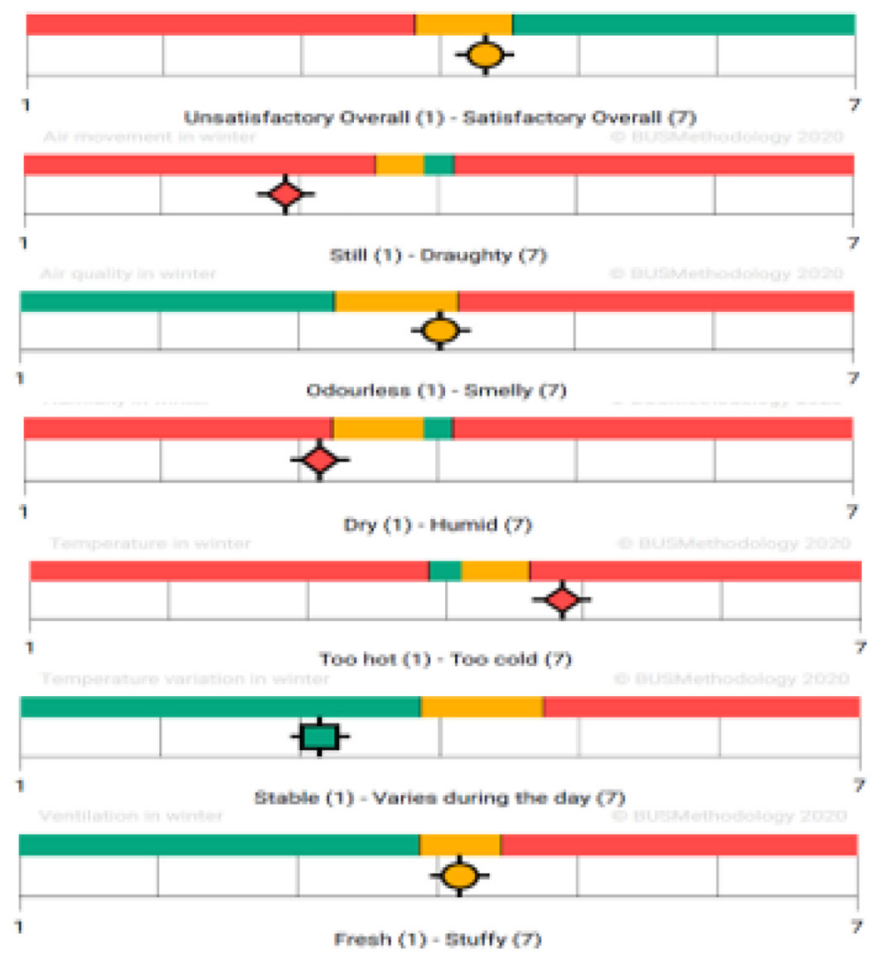

Fresh (1) - Sifufly (7)

Fig. 6. Question mean response of conditions in winter relative to the benchmark set.

The assessment results of temperature in winter are processed according to the following rules. Take the Rule 1 in Table 3 as an example. The evaluated non-domestic building (endb) has the evaluation task of temperature in winter (ttiw), this task is regulated by the criteria temperature in winter (ctiw) and, this criteria has a lower critical limit value (lclv) from the survey database; if the building occupants' question mean response value (qmrvtwhot) is less than the lower criterial limit value (lclv), then this evaluated non-domestic building (endb) will has a result with too hot.

\subsection{Implementation and evaluation using BUS methodology case study}

Building Use Studies (BUS) is a methodology for assessing occupant's satisfaction that was developed and refined during the 1990s, it has been used in over 850 buildings since its inception. The current non-domestic database has more than 850 buildings from around the world, and it has established a consistent dataset of quality resulting from over 70,000 building user responses. It is a paper-based and web-based questionnaire, and contains up to 45 quantitative and qualitative questions, and covers the aspects of thermal comfort, ventilation, indoor air quality, lighting, noise, space, personal control, image, needs, productivity and so on. Building occupants rate various performancerelated aspects on a scale of 1 to 7 , they are also allowed to provide written feedback [40]. The obtained feedback can be used to help identify the problems, and improve future quality and performance. By benchmarking occupant satisfaction levels against the established consistent dataset, the solutions can be found with informed decisions to improve occupant living experience and optimise performance. The BUS methodology has been widely used in POE research [41]. It is also a recognised tool for postoccupancy evaluation contributing towards BREEAM, LEED, WELL Standard, NABERS and Soft Landings.

As shown in Figure 6, the BUS methodology results for each question are reported using statistical tables, graphs and plots. Slider graphics with Red, Amber, Green (RAG) markers are used for ease of interpretation. Green rectangles represent survey mean values significantly better or higher than both the benchmark value. Amber ovals are survey mean values no different from the benchmark value. Red diamonds are survey mean values worse or lower than the benchmark value.

In this research, the BUS methodology has been adopted to carry out a field occupant satisfaction survey conducted in two non-domestic buildings. 150 paper questionnaires were handed out in each building, a total of 78 and 88 valid questionnaires were collected respectively from these two buildings, and the response rate was higher than $50 \%$. Part of the survey results are shown in Figure 6 , the conditions in winter overall satisfactory level is in the satisfied zone (amber and green zone), however, when it comes to the single assessment criteria, the values of temperature, humidity and air movement in winter are all in unsatisfactory zone (red zone). The assessment cannot find the problems if just checking the occupant satisfaction of conditions in winter overall, whereas the assessment from the single survey question can easily 
Table 4. Evaluation benchmark set of conditions in winter.

\begin{tabular}{lllllll}
\hline Items & & Mean & Percentile & SE & $\begin{array}{l}\text { Mean } \\
-\mathbf{1 . 9} \text { SE }\end{array}$ & $\begin{array}{l}\text { Mean } \\
+\mathbf{1 . 9} \text { SE }\end{array}$ \\
\hline \multirow{4}{*}{ Conditions in Winter Overall } & Question mean response & 4.32 & 44.65 & - & - & - \\
& Benchmark mean & 4.34 & 44.92 & 0.10 & 4.16 & 4.53 \\
& Scale midpoint & 4.00 & 37.50 & - & 3.82 & 4.18 \\
\multirow{2}{*}{ Temperature in Winter } & Question mean response & 4.84 & 72.43 & - & - & - \\
& Benchmark mean & 4.48 & 53.72 & 0.06 & 4.36 & 4.60 \\
& Scale midpoint & 4.00 & 16.53 & - & 3.88 & 4.12 \\
\hline
\end{tabular}

Table 5. The OWL properties.

\begin{tabular}{llllll}
\hline Object & Thresholds & Level & Data property & $\begin{array}{l}\text { Data } \\
\text { value }\end{array}$ & $\begin{array}{l}\text { Data } \\
\text { type }\end{array}$ \\
\hline Conditions in Winter Overall & $1 \leq \mathrm{tiw}<3.82$ & Too smelly & hasLowerCritialLimitValue & $=3.82$ & Xsd:float \\
& $4.53 \leq \mathrm{tiw} \leq 7$ & Satisfactory & hasUpperCritialLimitValue & $=4.53$ & Xsd:float \\
& $1 \leq \mathrm{tiw}<3.88$ & Too hot & hasLowerCritialLimitValue & $=3.88$ & Xsd:float \\
Temperature in winter(tiw) & $3.88 \leq \mathrm{tiw} \leq 4.60$ & Satisfactory & - & - & - \\
& $4.60<\mathrm{tiw} \leq 7$ & Too cold & hasUpperCritialLimitValue & $=4.60$ & Xsd:float \\
\hline
\end{tabular}

where $e n d b$ is the evaluated non-domestic building, tiw is temperature in winter, ciwo is the conditions in winter overall, and $p t i w$ is the permissioned values of tiw. After defining all the concepts and the OWL properties in ontology, the SWRL rules can be edited based on these pre-defined items, the SWRL rule examples are shown as in Table 3. Then the ontology model can perform assessment query based on edited rules.

categorize the satisfied or unsatisfied factors, so the building operation system manager or occupants themselves can easily adjust building operation.

Take the conditions in winter overall and temperature in winter assessment criteria as examples, the question's mean value of the selected building and the benchmark set value are shown in Table 4. According to the BUS methodology rules, the lower critical limit value is the lower value of the survey scale midpoint lower and the benchmark lower, in this case, the survey scale midpoint value lower, so the survey scale midpoint value has been chosen as the lower assessment thresholds value. The upper critical limit is the higher value of the survey scale midpoint upper and the benchmark upper, in this case, the benchmark mean value upper, so the survey scale midpoint value has been chosen as the upper assessment thresholds value. This means the lower or upper critical values are varied in each different survey questions. As shown in Table 4, for the assessment criteria of conditions in winter overall, the lower scale midpoint value has been taken as the lower critical limit value, which is 3.82; the upper benchmark mean value has been taken as the upper critical limit value, which is 4.53 . The same value selection rules are applied in the assessment criteria of temperature in winter and other survey criteria.

The evaluation benchmark set in Table 4 can be transferred into the assessment criteria's OWL properties in ontology, as shown in Table 5 .

\section{Implementation of occupant satisfaction assessment querying}

\subsection{Rules reasoning engine}

The SWRL rules and data facts need to be processed in a reasoning engine to transfer SWRL rules and relevant OWL knowledge to the rule engine, and to transfer the inferred rule engine knowledge back to OWL knowledge. In this paper, a user-driven and open-sourced rule engine called Drools is used, as shown in Figure 7, with the SWRL Drools tab is displayed in a window under the SWRL Editor in Protégé 5.5.0. The 'OWL+SWRL- $>$ Drools' button will transfer SWRL rules and relevant OWL knowledge to the rule engine, the 'Run Drools' button will run the rule engine, the 'Drools->OWL' button will transfer the inferred rule engine knowledge to OWL knowledge.

\subsection{Ontology querying evaluation}

As shown in Figure. 7, the rule engine shows the number of inferred axioms and the knowledge transfer process time. From the experimental results, the proposed assessment method has shown that compared to a manual assessment process, the proposed method can improve the efficiency of evaluation.

Figure 8 shows the translated inferred axioms to OWL knowledge. Take one of the evaluated buildings Michael 


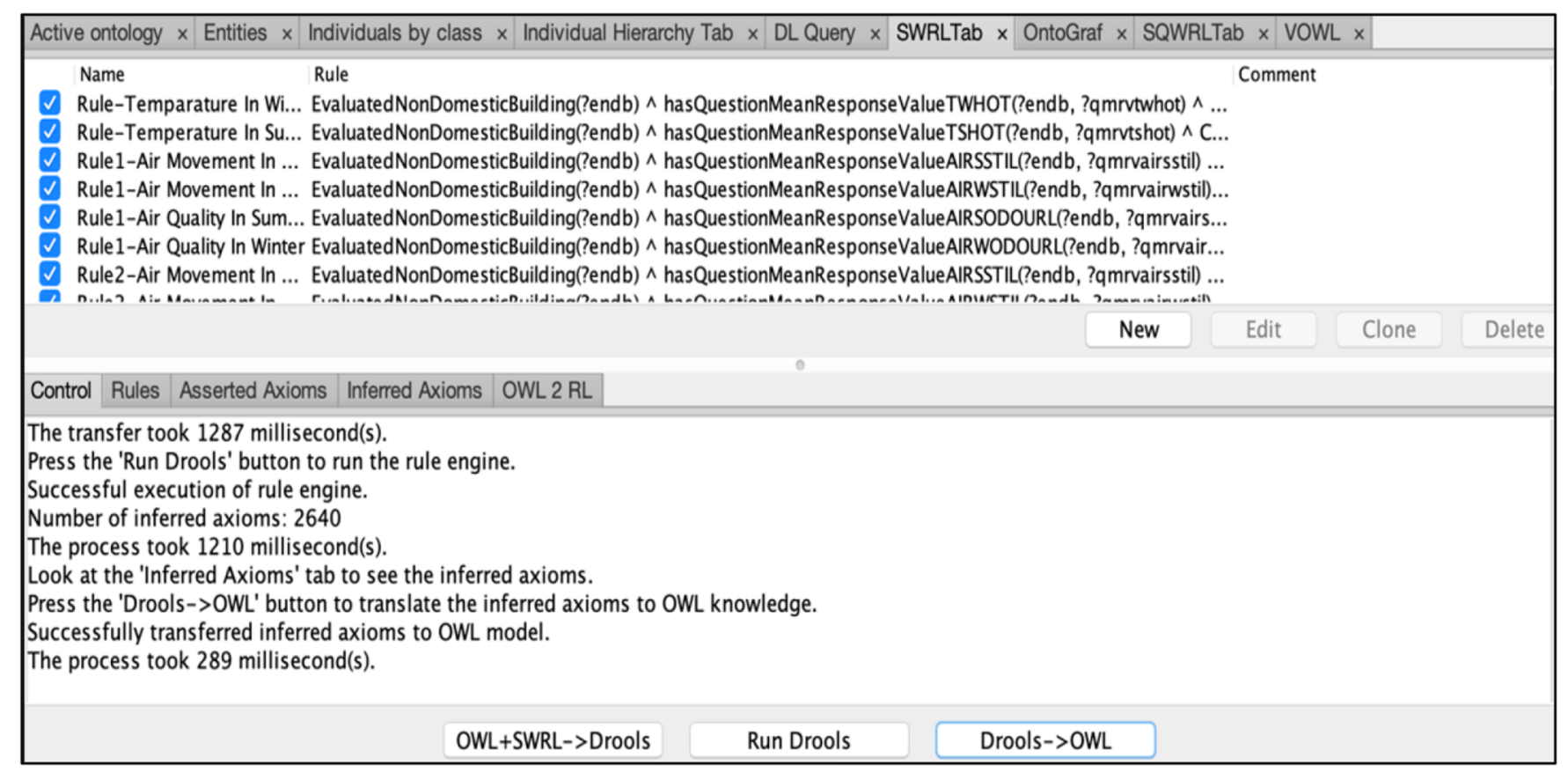

Fig. 7. Reasoning engine.

\begin{tabular}{|l|l|}
\hline MichaelSterlingBuilding & Property assertions: MichaeISt \\
\hline MultiFamilyHome & Object property assertions \\
Office & \\
Satisfactory & \\
Shop & Data property assertions \\
SingleFamilyHome & hasLowerCritial LimitValue \\
SouthernHallDormitory & ChasQuestionMeanRespons \\
\hline
\end{tabular}

(a)

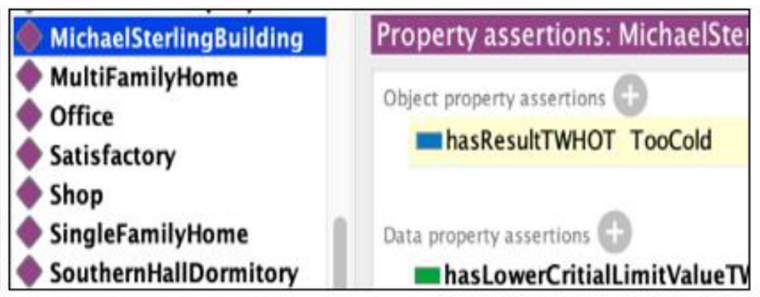

(b)

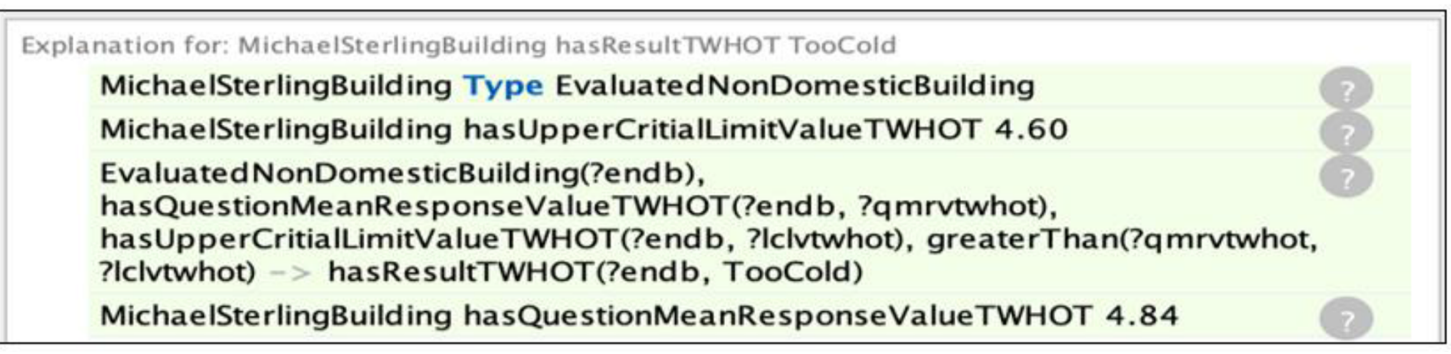

Fig. 8. Translate the inferred axioms to OWL knowledge (a) before reasoning. (b) after reasoning.

Sterling, as an example. Figure $8 \mathrm{a}$ is the initial OWL knowledge before reasoning, then based on the predefined SWRL reasoning rules, the inferred new knowledge after running the reasoning engine is highlighted in yellow automatically in Figure 8b. Figure 8c is the completed explanation of the inferred axioms. In this case, the criteria temperature in winter has two thresholds, the lower critical limit value of 3.88 and the upper critical limit value of 4.60 . If the evaluated value is greater than 4.60 , then the result is too cold; if the valuated value is less than 3.88 , then the result is too hot; if the evaluated value is between 3.88 and 4.60 , then the result is satisfactory. Here, the evaluated question mean response value of temperature in winter is 4.84 , which is greater than 4.60, so the evaluated building has the result of too cold.

The new knowledge generated from the logic reasoning process is translated into occupant satisfaction assessment results, and the building management system can then easily find the building operation problems, and adjust the operation plan based on the feedback results to improve the satisfaction level of the building. 


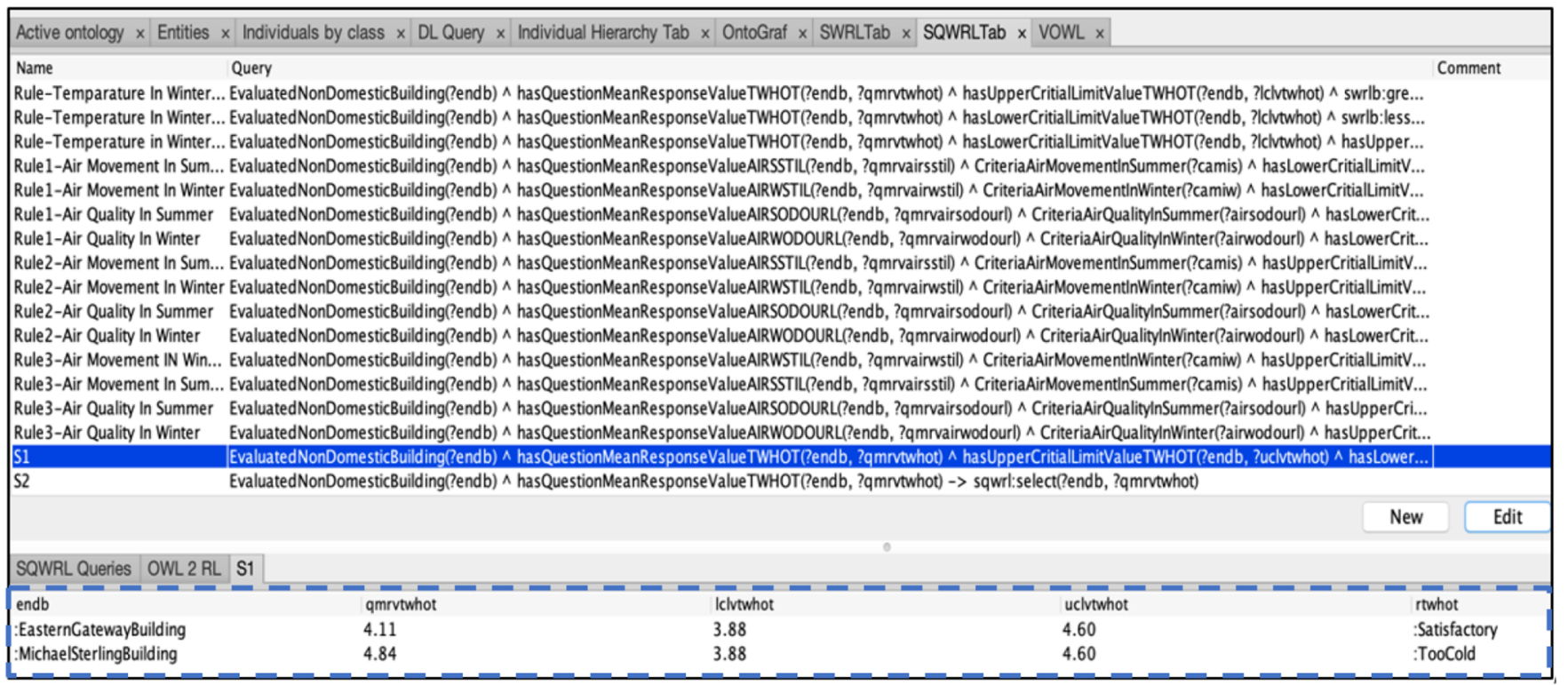

Fig. 9. Querying results.

After defining the SWRL rules in ontology model, the SQWRL tab in Protégé can be used to do assessment query based on edited rules. SQWRL takes a standard SWRL rule antecedent and effectively treats it as a pattern specification for a query. The core SQWRL operator is sqwrl:select. It contains one or more arguments, which are typically variables used in the pattern specification of the query, and builds a table using the arguments as the columns of the table [42]. For example, in this ontology model, the rule of querying the question mean response value, the lower and upper benchmarking thresholds, and the questionnaire survey result of the temperature in winter in a non-domestic building can be simply written as the follow query pattern:

EvaluatedNonDomesticBuilding(?endb $)^{\wedge}$ hasQuestionMeanResponseValueTWHOT(?endb, ?qmrvtwhot) $\wedge$ has UpperCritialLimitValueTWHOT(?endb, ? uclvtwhot) ${ }^{\wedge}$ has Lower Critial Limit Value TWHOT(? endb, ? lclvtwhot) ${ }^{\wedge}$ hasResultTWHOT(?endb, ?rtwhot)->sqwrl:select(?endb, ?qmrvtwhot, ?lclvtwhot, ?uclvtwhot, ?rtwhot).

Where qmrvtwhot is the question mean response value of temperature in winter, uclvtwhot is the upper critical limit value of temperature in winter, lclvtwhot is the upper critical limit value of temperature in winter, and rtwhot is the satisfactory result of temperature in winter.

The left side of the SQWRL query operator has the same form as the SWRL antecedent with its associated semantics. As shown in Figure 9, this query returns pairs of individuals and assessment values with one row for each pair. In this example, there are two individuals with their results examined by this query, one assessed building has the result with the satisfactory temperature in winter; another building with the result of too cold, which means the temperature in winter is not satisfied. The assessment process using the edited rules and queries is thus highly effective and easy to create, also clearly showing the thresholds values.

\section{Conclusions}

Post-occupancy evaluation (POE) is an effective method to evaluate the actual building performance after the building has been occupied for some time, it offers an opportunity to investigate the buildings' actual performance based on the occupants' satisfaction level in different aspects. Buildings are built to serve people, so it is important to understand their occupants' use experiences to optimize the building operation performance and improve the user experience, it is also a key part of POE. However, the occupant satisfaction assessment (OSA) can be easily ignored after the building has been delivered, and there is no systematic evaluation method. Moreover, it is time-consuming and error-prone to conduct the assessment manually.

The proposed post occupancy satisfaction evaluation system can be easily adapted to assess the occupant's satisfaction in a real-time approach. First, the BUS methodology is chosen as a field satisfaction survey tool to assess the occupant's satisfaction, its clauses are selected to validate the feasibility of the proposed approach, which are also the main conceptual structure of the developed ontology knowledge model. Second, the ontology technology is used to encapsulate domain knowledge of occupant satisfaction assessment in POE domain. Third, the benchmarking evaluation rules that are encoded in SWRL rules to enable automatic rule-based reasoning in a reasoning engine. The rule engine shows the number of inferred axioms and the knowledge transfer process time. Lastly, the query engine SQWRL is used to query the model. 
Based on the experimental results, the proposed assessment method in this research shows that compared to a manual assessment process, the proposed method improves the efficiency of evaluation. This ontology model also enables the effective OSA-related knowledge retrieving and sharing, and promotes its implementation. The POE ontology developed in this research can be easily upgraded to a new ontology based on changes in occupant satisfaction assessment benchmark database, and the SWRL rules can be easily updated as well. In short, this proposed framework separates the knowledge and the system functions, making it more flexible, easier to manage, share and retrieve the occupant satisfaction assessment knowledge in POE domain.

The authors would like to thank Adrian Leaman from BUS METHODOLOGY for sharing his valuable knowledge on using BUS methodology, and the anonymous reviewers who gave valuable suggestions that have helped to improve the quality of the manuscript.

\section{References}

1. X. Shi et al., Magnitude, causes, and solutions of the performance gap of buildings: a review, Sustainability 11, $1-21(2019)$

2. C.J. Roberts, D.J. Edwards, M.R. Hosseini, M. MateoGarcia, D.G. Owusu-Manu, Post-occupancy evaluation: a review of literature, Eng. Constr. Archit. Manag. 26, 2084-2106 (2019)

3. L.C. Guyot, Combining Post Occupancy Evaluation with the United States Green Building Council's Ledership in Energy and Environmental Design Rating System (2009)

4. J. Palmer, N. Terry, P. Armitage, Building Performance Evaluation Programme: findings from non-domestic projects Getting the best from buildings (2016)

5. A. Ratcliffe, A. Bateson, J. Hepburn, M. Savage, J. Warne, H. Lea, Soft Landings Framework-Xis Phases for Better Buildings BG 54/2018 (2018)

6. N. Khair, H.M. Ali, I. Sipan, N.H. Juhari, S.Z. Daud, Post occupancy evaluation of physical environment in public lowcost housing, J. Teknol. 75, 155-162 (2015)

7. N. Alborz, U. Berardi, A post occupancy evaluation framework for LEED certified U.S. higher education residence halls, Proc. Eng. 118, 19-27 (2015)

8. MOHURD, Technical manual of Post - occupancy Evaluation for Green Building (Edition for office and Store Buildings). China (2017), pp. 1-89

9. W.F.E. Preiser, U. Schramm, Intelligent office building performance evaluation, Facilities 20, 279-287 (2002)

10. F. Fantozzi, M. Rocca, An extensive collection of evaluation indicators to assess occupants' health and comfort in indoor environment, Atmosphere (Basel). 11 (2020)

11. W.F.E. Preiser, Continuous quality improvement through post-occupancy evaluation feedback, J. Corp. Real Estate 5, 42-56 (2002)

12. BSI, BS 8536-1:2015 Briefing for design and construction Part 1: Code of practice for facilities management (Buildings infrastructure), BSI Standards Publication (BSI Standards Limited, 2015), pp. 1-96
13. A. Blyth, A. Gilby, M. Barlex, Guide to Post Occupancy Evaluation, High. Educ. Funding Counc. Engl. (2006) p. 62

14. M.L. Pannier, C. Lemoine, M. Amiel, H. Boileau, C. Buhé, R. Raymond, Multidisciplinary post-occupancy evaluation of a multifamily house: an example linking sociological, energy and LCA studies, J. Build. Eng. 37 (2021)

15. S.K. Sansaniwal, J. Mathur, S. Mathur, Review of practices for human thermal comfort in buildings: present and future perspectives, Int. J. Ambient Energy 0, 1-27 (2020)

16. M.V. Bavaresco, S. D'Oca, E. Ghisi, R. Lamberts, Methods used in social sciences that suit energy research: a literature review on qualitative methods to assess the human dimension of energy use in buildings, Energy Build. 209, 109702 (2020)

17. T. Berners-Lee, J. Hendler, O. Lassila, The semantic web: a brain for humankind, Sci. Am. 284, 34-43 (2001)

18. T.R. Gruber, A translation approach to portable ontology specifications, Knowl. Acquis. 5, 199-220 (1993)

19. M. Uschold, M. Gruninger, Ontologies: principles, methods and applications, Knowl. Eng. Rev. 11, 93-136 (1996)

20. N.F. Noy, D.L. McGuinness, Ontology Development 101: A Guide to Creating Your First Ontology (2001)

21. A. Abadi, H. Ben-Azza, S. Sekkat, Improving integrated product design using SWRL rules expression and ontologybased reasoning, Proc. Comput. Sci. 127, 416-425 (2018)

22. C. Lork et al., An ontology-based framework for building energy management with IoT, Electronics 8, 485 (2019)

23. M. Bansal, J. Arora, A review on ontology based information retrieval system, Int. J. Eng. Dev. Res. 4, 2321-9939 (2016)

24. I. Automation, C. Technology, M. Bul, Ontology based data and information integration in biomedical, Mach. Technol. Mater. 3, 23-26 (2015)

25. Z. Zhou, Y.M. Goh, L. Shen, Overview and analysis of ontology studies supporting development of the construction industry, J. Comput. Civ. Eng. 30, 1-14 (2016)

26. M.J. Darlington, S.J. Culley, Investigating ontology development for engineering design support, Adv. Eng. Inf. 22, 112-134 (2008)

27. F.H. Abanda, J.H.M. Tah, Towards developing a sustainable building technology ontology, in Procs 24th Annual ARCOM Conference, edited by A. Dainty (2008) pp. 1-3

28. M. Kadolsky, K. Baumgärtel, R.J. Scherer, An ontology framework for rule-based inspection of eeBIM-systems, in Creative Construction Conference 2014 An (2014), vol. 85, pp. 293-301

29. C. Boje, Knowledge representation, storage and retrieval for BIM supported building evacuation design, Cardiff University (2018)

30. D. Zhang, J. Zhang, J. Guo, H. Xiong, A semantic and social approach for real-time green building rating in BIM-based design, Sustainable 11, 1-16 (2019)

31. L.Y. Ding, B.T. Zhong, S. Wu, H.B. Luo, Construction risk knowledge management in BIM using ontology and semantic web technology, Saf. Sci. 87, 202-213 (2016)

32. Y. Lu, Q. Li, Z. Zhou, Y. Deng, Ontology-based knowledge modeling for automated construction safety checking, Saf. Sci. 79, 11-18 (2015)

33. J. Xiao, X. Li, Z. Zhang, J. Zhang, Ontology-based knowledge model to support construction noise control in China, J. Constr. Eng. Manag. 144, 1-17 (2018)

34. C. Reinisch, M.J. Kofler, F. Iglesias, W. Kastner, Thinkhome energy efficiency in future smart homes, Eurasip J. Embed. Syst. 2011 (2011) 
35. Y. Zhao, Q. Yang, A. Fox, T. Zhang, Ontology-based knowledge modeling of post-occupancy evaluation for green building, in IOP Conference Series: Earth and Environmental Science (2020), vol. 495

36. D. Bonino, F. Corno, Ontology modeling for intelligent domotic environments, in 7th International Semantic Web Conference, ISWC2008 (2008) vol. 5318, pp. 790-803

37. W. Yan, C. Zanni-Merk, F. Rousselot, D. Cavallucci, P. Collet, Ontology-based knowledge modeling for using physical effects, Proc. Eng. 131, 601-615 (2015)

38. M. O'Connor et al., Using semantic Web technologies for knowledge-driven querying of biomedical data, Lect. Notes Comput. Sci. (including Subser. Lect. Notes Artif. Intell. Lect. Notes Bioinformatics), vol. 4594 LNAI (2007), pp. 267-276
39. M.J. O'Connor, A. Das, The SWRLTab: an extensible environment for working with SWRL Rules in Protege-OWL (2005). Available: https://protege.stanford.edu/conference/ 2007/slides/08.01_OConnor.pdf.

40. A. Leaman, BUS $\bar{S}$ occupant survey method: Details for licensees (2011)

41. J. Woo, A post-occupancy evaluation of a modular multiresidential development in Melbourne, Australia, in International High-Performance Built Environment Conference - a Sustainable Built Environment Conference 2016 Series (2017) vol. 180, pp. 365-372

42. M. O'Connor, A. Das, SQWRL: a query language for OWL, in CEUR Workshop Proceedings (2009), vol. 529, pp. $3-10$

Cite this article as: Yuanhong Zhao, Qingping Yang, Development of an ontology-based semantic building post-occupancy evaluation framework, Int. J. Metrol. Qual. Eng. 12, 19 (2021) 